\title{
Strut Onlay Allograft in Revision Arthroplasty: Osseous Union and Clinical Outcome
}

\author{
DIETMAR DAMMERER ${ }^{1}$, ALEXANDER WURM $^{1}$, CLEMENS FRISCHHUT $^{1}$, \\ JOHANNES PETERSEN ${ }^{2}$, MIAR OUARET ${ }^{2}$ and MICHAEL LIEBENSTEINER ${ }^{1}$ \\ ${ }^{1}$ Department of Orthopaedics and Traumatology, Medical University of Innsbruck, Innsbruck, Austria; \\ ${ }^{2}$ Department. of Radiology, Medical University of Innsbruck, Innsbruck, Austria
}

\begin{abstract}
Aim: To evaluate the clinical outcome and the osseous union of strut onlay allografts (SOAs) used as adjunct in revision total joint arthroplasty (TJA). Patients and Methods: Patients that had previously undergone SOA augmentation were considered for inclusion. Patients were invited to provide information for the following: Western Ontario and McMaster Universities Osteoarthritis Index (WOMAC), EuroQol five dimension score (EQ-5D) and Parker mobility score. Osseous union rates between SOA and the host bone were determined by radiograph with the Emerson classification system. Bone mineral density was measured via quantitative computed tomography. Results: Seventeen patients were identified, at a mean follow-up of 2.8 years. The median total WOMAC score was 22 [interquartile range $(I Q R)=21$ ]. The median $E Q-5 D$ score was 0.887 (IQR=0.350) (time trade-off). The Parker Mobility Score was $8.0(I Q R=3.5)$. Emerson stages of radiographic graft to host union were 'rounding off' in one case, 'partial bridging' in three and 'complete bridging' in 13. Quantitative computed tomography showed an average bone mineral density of approximately $1,300 \mathrm{mg} / \mathrm{cm}^{3}$. Conclusion: From our findings, it is concluded that SOAs used in revision total joint arthroplasty provide promising results and are recommended for broader clinical use. A complete osseous union between host and graft bone was observed in the majority of cases.
\end{abstract}

This article is freely accessible online.

Correspondence to: Alexander Wurm, MD, Department of Orthopaedics and Traumatology, Medical University of Innsbruck, Anichstrasse 35, 6020 Innsbruck, Austria. Tel: +43 51250422691 , Fax: +43 51250422693, e-mail: alexander.wurm@i-med.ac.at

Key Words: Revision arthroplasty, strut graft, strut allograft, strut onlay allograft, quantitative computed tomography, bone mineral density.
Severe bone deficiencies of the femur or tibia can occur after revision surgery in total hip or knee arthroplasty, tumor resections and in complicated fractures (e.g., periprosthetic fractures). To manage these defects, strut allografts are frequently used (1).

Vascularized or non-vascularized strut autografts (e.g., fibula) were reported quite often for augmentation of such bony defects but are also associated with severe donor site morbidity (2-10). The literature reports the use of strut onlay allografts (SOAs) (e.g., part of a fresh-frozen femoral diaphysis) as a reliable alternative for bone augmentation instead of vascularized or non-vascularized strut autografts (11-13). SOAs are individually sized beams of non-vital bone attached to the deficient femur via tension or cable wires (1416). Incorporation and remodeling of these allografts to the host bone has been shown to be successful in $80-100 \%$ of cases (1). Although primary stability is the basic requirement for osseointegration and subsequent good long-term stability of the implant (1-10), there is no evidence that strut grafts have an effect on primary fixation of revision stems. To the best of our knowledge, such reports are rare and we are aware of only very few clinical reports on the union rate and clinical outcome of SOAs in revision arthroplasty (17-19).

We therefore aimed to perform a retrospective investigation of patients previously treated with SOAs at our department. We assume i) high union rates between SOAs and host bone, ii) good clinical outcome as determined from patient-reported outcome, and iii) little or no loss of correction/alignment.

\section{Patients and Methods}

The local Institutional Review Board approved the study protocol (registry number: AN2016-0023 358/4.12). Informed consent was obtained from all participants before participation in our study.

We identified and included all consecutive patients who received a fresh-frozen SOA at our Institution between 2004 and 2017. Data were collected retrospectively from our Bone Bank Database and patients' medical records. We included all patients with a) a 
Table I. Patients included, indication and treatment.

\begin{tabular}{|c|c|c|}
\hline Indication & Treatment & Recipient bone \\
\hline \multirow[t]{2}{*}{ Periprosthetic femoral fracture in THA $(n=7)$} & $\begin{array}{l}\text { Replacement of the femoral stem and SOA augmentation }(n=3) \\
\text { Plate osteosynthesis and SOA augmentation }(n=2)\end{array}$ & \\
\hline & Fixation of the SOA with $2.0-\mathrm{mm}$ cables exclusively $(\mathrm{n}=2)$ & Femur \\
\hline Aseptic loosening of the THA $(n=4)$ & Change of the femoral stem and SOA augmentation & Femur \\
\hline Periprosthetic tibial fracture in TKA $(n=1)$ & Change of the TKA and SOA augmentation & Tibia \\
\hline Aseptic loosening of the TKA $(n=1)$ & Change of the TKA and SOA augmentation & Femur \\
\hline Recurrent THA luxations $(\mathrm{n}=1)$ & Change of the femoral stem for malalignment and SOA augmentation & Femur \\
\hline Bone deficits due to tumor resections $(n=1)$ & Change of the mega-prosthesis and SOA augmentation & Femur \\
\hline Bone deficiency after THA $(n=1)$ & Revision of the THA and SOA augmentation & Femur \\
\hline Bone deficiency after TKA $(n=1)$ & Revision of the TKA and SOA augmentation & Femur \\
\hline
\end{tabular}

SOA: Strut onlay allograft; THA: total hip arthroplasty; TKA: total knee arthroplasty.

condition after revision hip or knee arthroplasty induced by inadequate osseous union of the hip, or knee replacement (pseudarthrosis); b) a condition after periprosthetic femoral or tibial fracture; c) a femoral or tibial bone augmentation associated with bone deficits due to massive tumor resection; or d) a femoral or tibial bone augmentation associated with bone deficiency due to prosthetic implantation. In all investigated cases, a fresh-frozen SOA from a femoral diaphysis allograft was applied. The strut was cut out of a whole femoral diaphysis in a way to best fit the shape of the recipient bone.

Patients were contacted and invited to undergo examinations after we obtained informed consent. Patient inclusion criteria are shown in Table I. We assessed the patient-reported clinical outcome with the Western Ontario and McMaster Universities Osteoarthritis Index (WOMAC) (20), EuroQol five dimension (EQ-5D) score (21) and the Parker Mobility Score (22). The WOMAC questionnaire consists of the subscales WOMAC pain (five items), WOMAC stiffness (two items) and WOMAC functional limitation (17 items), which are totaled and expressed as percentages of the maximum score of the subscales. All 24 items were standardized to a range of 0 (none) to 100 (worst). Additionally, the three subscales were totalled and also expressed as percentages (WOMAC Global Score). The higher the score, the greater the pain and stiffness and the higher the level of functional limitations.

In addition to the limb-specific outcome, the 'health-related quality of life' was assessed with the EQ-5D scale in a self-reported way (21). The questionnaire comprises five dimensions of health: Mobility, selfcare, usual activities, pain/discomfort, and anxiety/depression. The response possibilities for each of the five dimensions are: no problems, some problems and extreme problems, which result in 243 potential quality-of-life conditions. The standardized key metric, time trade-off (TTO), with a range from 0 (worst) to 1 (best), was used to assess the health-related quality of life. However, the EQ-5D questionnaire includes a visual analog scale (VAS) that shows the respondent's selfrated health status on a graduated $(0-100)$ scale, with higher scores for higher health-related quality of life.

Ambulatory status was assessed with the Parker Mobility Score $(22,23)$. The score includes three questions, each rated $0-3$ points Calculated from the total mobility assessment in three different situations (able to get about the house, able to get out of the house, and able to go shopping), the total score ranges from 0-9. The highest overall score of 9 indicates the best possible mobility (22).
Osseous union between the strut allograft and the host bone was verified by two independent radiologists and co-authors using two plain x-rays according to the classification system of Emerson et al. (6) : Rounding off, scalloping, partial bridging, complete bridging, cancellation and resorption. 'Loss of correction' was determined from the radiographs, by the same radiologists and was defined as current axial deviation as compared to the full-leg postoperative radiographs as a categorized variable $\left(0-5^{\circ}, 5-10^{\circ},>10^{\circ}\right)$.

SOA quality was also assessed with quantitative computed tomography (QCT) performed with a GE Discovery CT750 HD Scanner (GE, Milwaukee, WI, USA) using a standardized protocol (120 KV, mA Auto, $8.8 \mathrm{~s} / \mathrm{HE} ; 1.25 \mathrm{~mm}$ slice thickness) without radiopaque material. QCT was suggested in previous research and its findings are strongly correlated with mechanical stability (6). Bone mineral density (BMD) was calculated from three different circular volumes of interest (VOI) along the SOA (proximal, intermediate, and distal) as shown in Figure 1 using the PACS software Impax EE (Agfa HealthCare N.V., Mortsel, Belgium). Calculation of the BMD $\left(\mathrm{mg} / \mathrm{cm}^{3}\right)$ was performed with the European Forearm Phantom as described in previous studies (2428). It was reported that QCT exposes the patient to a minimal radiation dose if appendicular bones are investigated $(<1 \mu \mathrm{Sv})$.

Descriptive statistics were calculated as medians and means. Interquartile ranges (IQR) for numeric data and frequencies were used for ordinal data (medians) and ranges (min. to max.; means). SPSS, version 24.0 (IBM, Armonk, NY, USA) was used to evaluate the data. Microsoft Excel 2011, version 14.3.9 (Microsoft, Redmond, WA, USA) was used to create tables and diagrams. Ordinal data are presented as absolute or relative frequencies. The median and IQR were chosen to represent numerical data. The IQR was calculated using a weighted average. The IQR in our study was very symmetrical around the median, therefore we decided to report the IQR as a value instead of a range. In the graphic representations of the medians (box plot, bar charts, line charts), the IQR was defined using Tukey's hinges.

\section{Results}

From October 2004 to January 2017, a total of 49 SOAs were found in our bone bank register. Of these 49 patients, $13 \mathrm{had}$ died (not related to the surgery) during the follow-up period and 15 further patients either could not be found or 


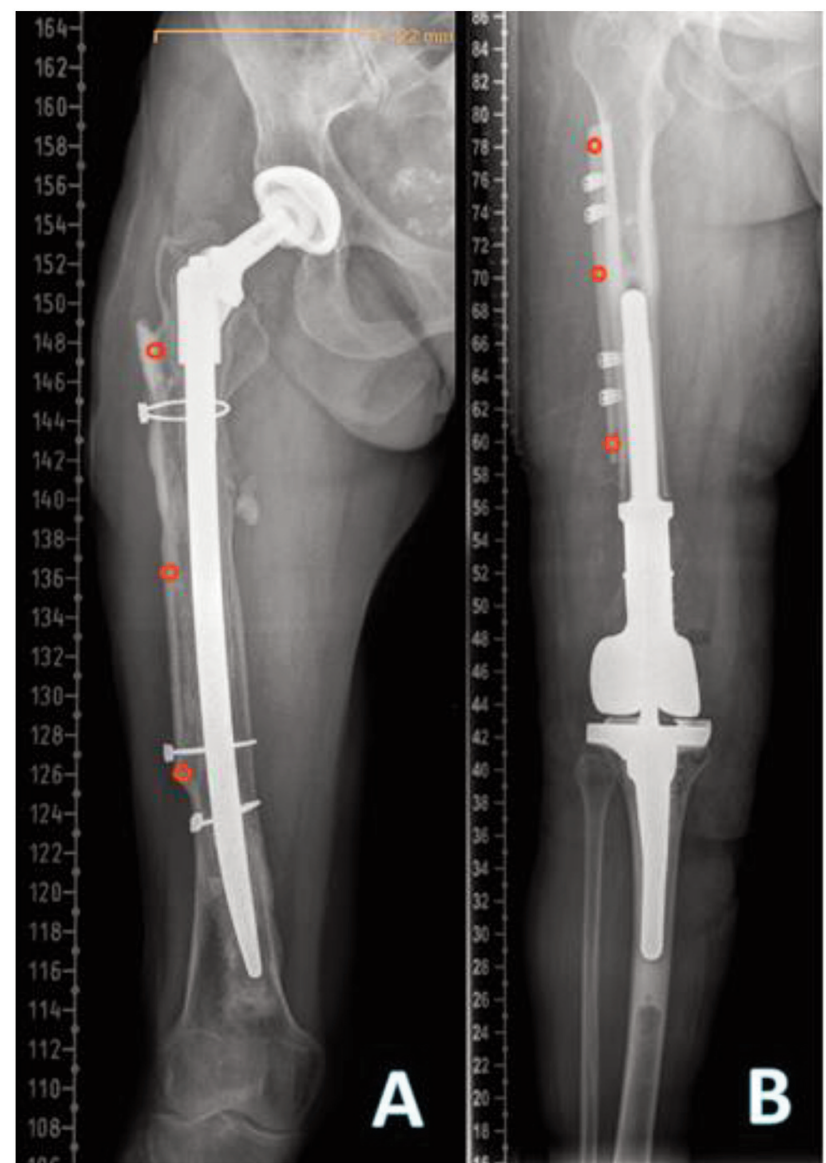

Figure 1. Bone mineral density was calculated in three different circular regions of interest along the strut onlay allograft, from proximal, intermediate to distal. Regions of interest were marked with a red circle along the femur in a revision arthroplasty of the hip (A) and revised knee replacement $(B)$. In our Department, we prefer to use wires for fixation of the strut onlay allograft.

refused to participate. We had to exclude another three patients because they did not receive a SOA on the femoral or tibial site. In addition, one patient had to be excluded due to a stroke during the follow-up. A total of 17 SOAs in 17 patients of whom five were male and 12 female, at a mean age of $66.0 \pm 11.9$ years mean height of $164.0 \pm 15.5 \mathrm{~cm}$ and a mean body mass index of $23.2 \pm 7.3 \mathrm{~kg} / \mathrm{m}^{2}$ were included in our study. Details are shown in the flow chart (Figure 2).

The mean age at SOA implantation was $68($ range $=20-83)$ years. Mean body mass index was $23.2 \pm 7.3 \mathrm{~kg} / \mathrm{m}^{2}$. Mean Charlson comorbidity index was 6.9 (range=2-10). Mean American Society of Anesthesiologists score was 2.6 (range=2-4). Mean follow-up was 2.8 (range=0.5-12.0) years. A total of 13 total hip arthroplasties, three total knee arthroplasties and one tumor resection were included. Sixteen SOAs were implanted on the femur and one on the tibia. Details concerning indication are shown in Table I.
In our study group, we recorded three out of the six possible SOA healing stages according to Emerson et al. (6): In one patient, the SOA showed rounding off, in three it showed partial bridging, and in 13 complete bridging. No scalloping, cancellation, or resorption condition was found in the examined patients.

Sixteen SOAs showed a loss of correction of $0-5^{\circ}$ (i.e., no or minimal loss of correction). An axial deviation of $5-10^{\circ}$ was observed in one case. Closer analysis of the radiographs of that particular case revealed that most likely the failure was not related to the strut allograft itself but rather to the surgical technique. At the time of the observed loss of correction of $5-10^{\circ}$, the service life of the SOAs was 1 year, the patient's age at the time of surgery was 72 years.

BMD was calculated from three VOIs along the SOA. The median BMD was $1,226(\mathrm{IQR}=166) \mathrm{mg} / \mathrm{cm}^{3}$ for VOI 1 (proximal), 1,319 $(\mathrm{IQR}=278) \mathrm{mg} / \mathrm{cm}^{3}$ for VOI 2 (intermediate) and $1,330(\mathrm{IQR}=257) \mathrm{mg} / \mathrm{cm}^{3}$ for VOI 3 (distal).

The median total WOMAC score was $22(\mathrm{IQR}=21)$. The median score for WOMAC pain was $16(\mathrm{IQR}=23)$, for WOMAC stiffness was $15(\mathrm{IQR}=28)$ and for WOMAC function was $25(\mathrm{IQR}=32)$ The median $\mathrm{EQ}-5 \mathrm{D}$ score by TTO was 0.887 (IQR=0.350) and by VAS was $0.658(\mathrm{IQR}=0.490)$. The Parker Mobility Score was 8.0 (IQR=3.5).

\section{Discussion}

The most important findings of our study are that a) there was a beneficial rate of fusion between the SOA and the recipient bone, with 13 patients showing complete fusion and three patients partial fusion, and b) these had clinically successful results as shown by patient-reported outcomes WOMAC, EQ5D quality-of-life score and Parker Mobility Score.

When comparing our findings with those of others, it appears that only three previous studies investigated the union rates and the clinical outcome of SOAs in the field of total joint arthroplasty reoperation (14-16). Yeo et al. retrospectively reviewed 17 cases treated with the combination of a locking plate and anterior SOA for periprosthetic hip fractures (14). In 39 patients, after a mean follow-up of 28 months, the authors reported that $88 \%$ of the patients had good or excellent results in Harris Hip Score and a fracture healing rate of $100 \%$. Similarly to our study, Yeo et al. found only 'complete bridging' (nine patients)' and 'partial bridging' (eight patients) Emerson stages in their population. Leino et al. retrospectively analyzed patients with supracondylar periprosthetic fractures after knee arthroplasty (15). Among their patients, 39 were treated with internal fixation. Of those, 33 additionally received a SOA. Of their patients, 29 were treated with revision arthroplasty, five of whom additionally received a SOA. The authors reported a positive clinical outcome in $88 \%$ (31 out of 39 patients). In the osteosynthesis group, 1-year implant survival was $50 \%$ in 


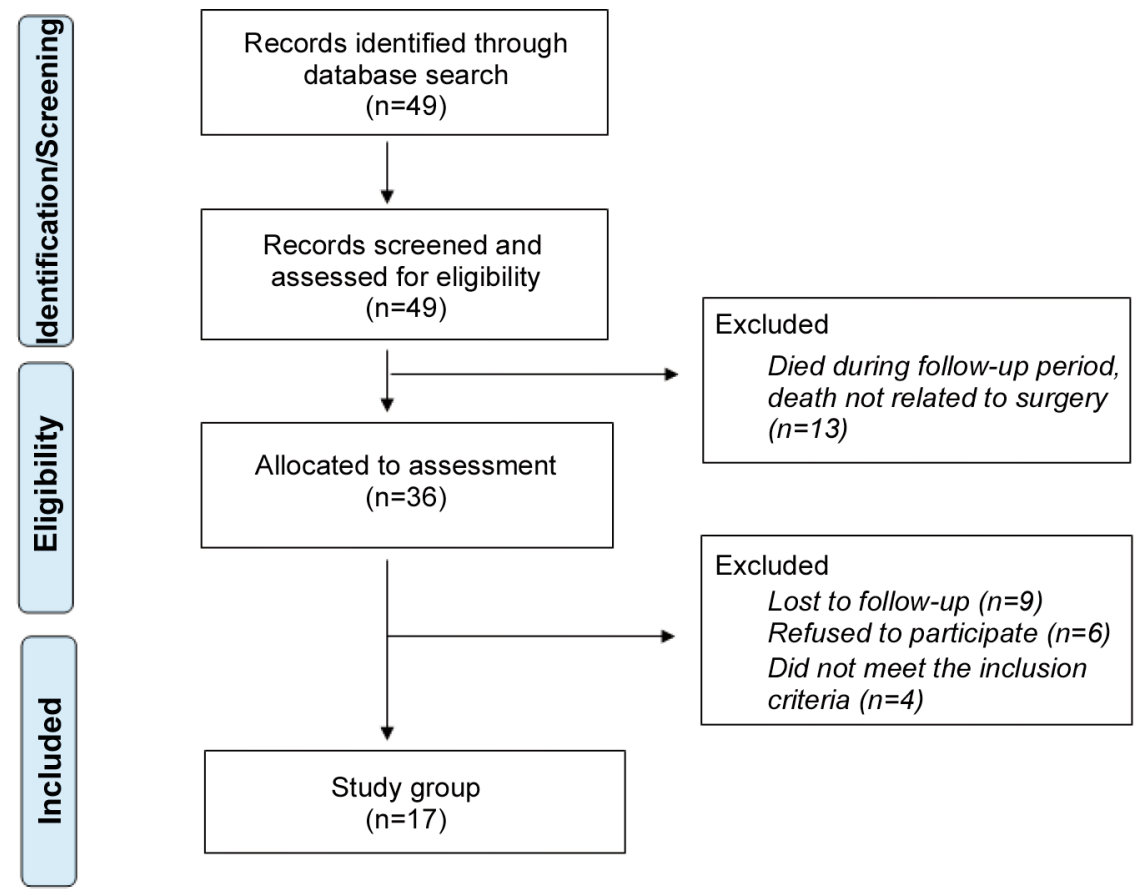

Figure 2. Bone mineral density was calculated in three different circular regions of interest along the strut onlay allograft, from proximal, intermediate to distal. Regions of interest were marked with a red circle along the femur in a revision arthroplasty of the hip (A) and revised knee replacement (B). In our Department, we prefer to use wires for fixation of the strut onlay allograft.

those with stand-alone plating and $80 \%$ in those with an additional SOA. The study by Akiyama et al. presented data on 21 patients that received an SOA for a variety of indications in the field of arthroplasty (stem loosening, periprosthetic fracture, infection) (16). The authors reported no cases that clinically failed. On average, partial host-graft union was noted after 41 weeks and total union was noted 82 weeks postoperatively.

In summary, previous research provides only scarce evidence of union rates and clinical outcome of SOAs in revision arthroplasty. However, the available evidence from each of the previous three studies is promising. Those promising results are highly congruent with the findings of the current study, which also found favorable WOMAC scores, good quality of life, good ambulatory status, and improved radiographic fusion rates. What is different between the three former studies and ours is that we also analyzed tissue quality by means of QCT (BMD). The BMD was determined to be approximately $1,300 \mathrm{mg} / \mathrm{cm}^{3}$ in the SOA, thus falling in the typical range for healthy cortical bone (29). Previous research suggested that BMD be measured with QCT, and the findings strongly correlated with mechanically stable bones.

When also considering in vitro studies on SOAs, there is evidence from Sariyilmaz et al., who analyzed the effect of additional SOAs during internal fixation of Vancouver B1 periprosthetic hip fractures (30). The authors reported superior results for the SOA groups regarding cyclic axial and rotational loading simulations. Similar findings come from the cadaveric study by Wilson et al. (31). The authors tested plate only, SOA only, and plate-SOA combinations in the setting of periprosthetic fractures. From their findings obtained in rotational and axial laboratory tests, the authors concluded that the best mechanical properties were achieved when an SOA was added to a plate. Peters et al. also tested SOAs in a cadaveric study setting (32). The authors tested the stability of periprosthetic femur fractures fixed with either a plate or an SOA. Cadaveric femur specimens were loaded in single-leg stance and stair-climbing simulations. From their findings, the authors concluded that SOAs are a biomechanically sound alternative to metal plates. Conflicting findings were published by Dennis et al. (33). They used a periprosthetic fracture model with synthetic femora and different fixation options. During biomechanical testing simulations, the SOA fixed with cables was found to be inferior to plate-screw constructs. However, the authors did not investigate SOA-plate combinations. O'Connell et al. also conducted an in-vitro investigation of SOA use in a periprosthetic fracture model (34). The authors used artificial femora and applied three different types of Vancouver $\mathrm{C}$ fracture fixation: Plate only, plate with medial SOA, plate with anterior SOA. From their findings, the authors did not find additional value for the plate-SOA combination. This 
was because the authors regarded all three types of fixation as sufficient for assisted weight-bearing conditions. Potentially the authors did not consider geriatric settings with patients not being able to apply partial weight bearing.

Beyond the issue of fracture healing and primary stability the concept of the SOA may also be favorable in terms of adding bone stock and thus providing advantages for future revisions.

The following limitations of our study are acknowledged. Firstly, limitations are due to the retrospective study design and it is regarded as a shortcoming that many patients treated with a SOA were not available for examination. Of 49 patients in our bone bank registry, 13 had died (not related to their surgery), 15 patients either could not be successfully contacted or refused to participate and a further four patients did not fulfill the inclusion criteria, which left only 17 patients for the current investigation. Another limitation is the fact that the patients were operated on by a variety of surgeons at our Institution. It is regarded as a strength of our study that the 17 patients were assessed with clinically validated patient-reported outcomes. In addition, radiographic evaluation of bone union and measurement of the bone mineral density of the graft bone was obtained.

From our findings, it is concluded that SOAs used in revision total joint arthroplasty provide promising results. A complete osseous union between host and graft bone was observed in the majority of cases. Moreover, BMD values equaled what previous researchers found in healthy cortical bone. Favorable findings for ambulatory status, quality of life, and WOMAC scores further underline the procedure's potential. The SOA method is recommended for broader clinical use in revision arthroplasty.

\section{Conflicts of Interest}

None.

\section{Authors' Contributions}

D. Dammerer: Literature research, data analysis, editing, writing and proofreading of the article. A. Wurm: Study protocol, study design, literature research, data analysis, editing and writing of the article. C. Frischut: Data analysis and proofreading of the article. M. Ouaret: analyzing of radiographic images, proofreading of article. J. Peterson: Study protocol, study design, analyzing of radiographic images, and proofreading of article. M. Liebensteiner: Co-editing and proofreading of the article. All Authors made pertinent contributions to the article, and proofread and approved the final article before submission.

\section{Acknowledgements}

This study was carried out with internal funds from Department of Orthopaedics and Traumatology, Medical University of Innsbruck and Institute of Radiology, Medical University of Innsbruck.

\section{References}

1 Kinkel S, Thomsen MN, Nadorf J, Heisel C, Tanner MC and Jakubowitz E: Strut grafts in revision hip arthroplasty faced with femoral bone defects: an experimental analysis. Int Orthop 38(6): 1147-1153, 2014. PMID: 24384941. DOI: 10.1007/ s00264-013-2257-y

2 Allan DG, Lavoie GJ, McDonald S, Oakeshott R and Gross AE: Proximal femoral allografts in revision hip arthroplasty. J Bone Joint Surg Br 73(2): 235-240, 1991. PMID: 2005146. DOI: 10.1302/0301-620X.73B2.2005146

3 Barden B, Fitzek JG, Huttegger C and Löer F: Supportive strut grafts for diaphyseal bone defects in revision hip arthroplasty. Clin Orthop Relat Res (387): 148-155, 2001. PMID: 11400876. DOI: 10.1097/00003086-200106000-00020

4 Buoncristiani AM, Dorr LD, Johnson C and Wan Z: Cementless revision of total hip arthroplasty using the anatomic porous replacement revision prosthesis. J Arthroplasty 12(4): 403-415, 1997. PMID: 9195316. DOI: 10.1016/s0883-5403(97)90196-0

5 Chandler HP, Ayres DK, Tan RC, Anderson LC and Varma AK: Revision total hip replacement using the S-ROM femoral component. Clin Orthop Relat Res (319): 130-140, 1995. PMID: 7554622 .

6 Emerson RH Jr, Malinin TI, Cuellar AD, Head WC and Peters PC: Cortical strut allografts in the reconstruction of the femur in revision total hip arthroplasty. A basic science and clinical study. Clin Orthop Relat Res (285): 35-44, 1992. PMID: 1446451.

7 Gross AE, Blackley H, Wong P, Saleh K and Woodgate I: The role of allografts in revision arthroplasty of the hip. Instr Course Lect 51: 103-113, 2002. PMID: 12064094.

8 Head WC and Malinin TI: Results of onlay allografts. Clin Orthop Relat Res (371): 108-112, 2000. PMID: 10693555. DOI: 10.1097/00003086-200002000-00012

9 Kim YH and Kim JS: Revision hip arthroplasty using strut allografts and fully porous-coated stems. J Arthroplasty 20(4): 454-459, 2005. PMID: 16124960. DOI: 10.1016/j.arth. 2004.09.054

10 Pak JH, Paprosky WG, Jablonsky WS and Lawrence JM: Femoral strut allografts in cementless revision total hip arthroplasty. Clin Orthop Relat Res (295): 172-178, 1993. PMID: 8403644.

11 Qu H, Guo W, Yang R, Tang X, Yan T, Li D, Yang Y and Zang $\mathrm{J}$ : Cortical strut bone grafting and long-stem endoprosthetic reconstruction following massive bone tumour resection in the lower limb. Bone Joint J 97-B(4): 544-549, 2015. PMID: 25820896. DOI: 10.1302/0301-620X.97B4.34695

12 Rodríguez-Vegas JM and Delgado-Serrano PJ: Corticoperiosteal flap in the treatment of nonunions and small bone gaps: technical details and expanding possibilities. J Plast Reconstr Aesthet Surg 64(4): 515-527, 2011. PMID: 20678976. DOI: 10.1016/j.bjps.2010.06.035

13 Lauthe O, Soubeyrand M, Babinet A, Dumaine V, Anract P and Biau DJ: The indications and donor-site morbidity of tibial cortical strut autografts in the management of defects in long bones. Bone Joint J 100-B(5): 667-674, 2018. PMID: 29701102. DOI: 10.1302/0301-620X.100B5.BJJ-2017-0577.R2

14 Yeo I, Rhyu KH, Kim SM, Park YS and Lim SJ: High union rates of locking compression plating with cortical strut allograft for type B1 periprosthetic femoral fractures. Int Orthop 40(11): 2365-2371, 2016. PMID: 26759257. DOI: 10.1007/s00264-0153107-x 
15 Leino OK, Lempainen L, Virolainen P, Sarimo J, Pölönen T and Mäkelä KT: Operative results of periprosthetic fractures of the distal femur in a single academic unit. Scand J Surg 104(3): 200207, 2015. PMID: 25332221. DOI: 10.1177/1457496914552343

16 Akiyama H, Hachiya Y, Otsuka H, Kurisuno M, Kawanabe K, Katayama N, Ohura H, Yamamoto K, Sato K and Matsuda S: Low-intensity pulsed ultrasound therapy stimulates callus formation between host femur and cortical onlay strut allograft. Ultrasound Med Biol 40(6): 1197-1203, 2014. PMID: 24556560. DOI: 10.1016/j.ultrasmedbio.2013.12.030

17 Ducheyne P, De Meester P and Aernoudt E: Influence of a functional dynamic loading on bone ingrowth into surface pores of orthopedic implants. J Biomed Mater Res 11(6): 811-838, 1977. PMID: 591524. DOI: 10.1002/jbm.820110603

18 Engh CA, Bobyn JD and Glassman AH: Porous-coated hip replacement. The factors governing bone ingrowth, stress shielding, and clinical results. J Bone Joint Surg Br 69(1): 4555, 1987. PMID: 3818732. DOI: 10.1302/0301-620X.69B1. 3818732

19 Pilliar RM, Lee JM and Maniatopoulos C: Observations on the effect of movement on bone ingrowth into porous-surfaced implants. Clin Orthop Relat Res (208): 108-113, 1986. PMID: 3720113.

20 Bellamy N, Buchanan WW, Goldsmith CH, Campbell J and Stitt LW: Validation study of WOMAC: a health status instrument for measuring clinically important patient relevant outcomes to antirheumatic drug therapy in patients with osteoarthritis of the hip or knee. J Rheumatol 15(12): 1833-1840, 1988. PMID: 3068365.

21 EuroQol Group: EuroQol-a new facility for the measurement of health-related quality of life. Health Policy 16(3): 199-208, 1990. PMID: 10109801. DOI: 10.1016/0168-8510(90)90421-9

22 Parker MJ and Palmer CR: A new mobility score for predicting mortality after hip fracture. J Bone Joint Surg Br 75(5): 797-798, 1993. PMID: 8376443. DOI: 10.1302/0301-620X.75B5.8376443

23 Voeten SC, Nijmeijer WS, Vermeer M, Schipper IB, Hegeman JH and DHFA Taskforce study group: Validation of the Fracture Mobility Score against the Parker Mobility Score in hip fracture patients. Injury 51(2): 395-399, 2020. PMID: 31668574. DOI: 10.1016/j.injury.2019.10.035

24 Bottlang M, Lesser M, Koerber J, Doornink J, von Rechenberg B, Augat P, Fitzpatrick DC, Madey SM and Marsh JL: Far cortical locking can improve healing of fractures stabilized with locking plates. J Bone Joint Surg Am 92(7): 1652-1660, 2010. PMID: 20595573. DOI: 10.2106/JBJS.I.01111

25 Augat P, Merk J, Genant HK and Claes L: Quantitative assessment of experimental fracture repair by peripheral computed tomography. Calcif Tissue Int 60(2): 194-199, 1997. PMID: 9056170. DOI: 10.1007/s002239900213

26 Firoozabadi R, Morshed S, Engelke K, Prevrhal S, Fierlinger A, Miclau T 3rd and Genant HK: Qualitative and quantitative assessment of bone fragility and fracture healing using conventional radiography and advanced imaging technologiesfocus on wrist fracture. J Orthop Trauma 22(8 Suppl): S83-S90, 2008. PMID: 18753895. DOI: 10.1097/BOT.0b013e31815ea2a4
27 Reeve J, Kröger H, Nijs J, Pearson J, Felsenberg D, Reiners C, Schneider P, Mitchell A, Ruegsegger P, Zander C, Fischer M, Bright J, Henley M, Lunt M and Dequeker J: Radial cortical and trabecular bone densities of men and women standardized with the European Forearm Phantom. Calcif Tissue Int 58(3): 135143, 1996. PMID: 8852567. DOI: 10.1007/BF02526878

28 Krappinger D, Roth T, Gschwentner M, Suckert A, Blauth M, Hengg $\mathrm{C}$ and Kralinger F: Preoperative assessment of the cancellous bone mineral density of the proximal humerus using CT data. Skeletal Radiol 41(3): 299-304, 2012. PMID: 21509434. DOI: $10.1007 / \mathrm{s} 00256-011-1174-7$

29 Evans RK, Negus C, Antczak AJ, Yanovich R, Israeli E and Moran DS: Sex differences in parameters of bone strength in new recruits: beyond bone density. Med Sci Sports Exerc 40(11 Suppl): S645-S653, 2008. PMID: 18849870. DOI: 10.1249/ MSS.0b013e3181893cb7

30 Sariyilmaz K, Dikici F, Dikmen G, Bozdag E, Sunbuloglu E, Bekler B and Yazicioglu O: The effect of strut allograft and its position on Vancouver type B1 periprosthetic femoral fractures: a biomechanical study. J Arthroplasty 29(7): 1485-1490, 2014. PMID: 24656637. DOI: 10.1016/j.arth.2014.02.017

31 Wilson D, Frei H, Masri BA, Oxland TR and Duncan CP: A biomechanical study comparing cortical onlay allograft struts and plates in the treatment of periprosthetic femoral fractures. Clin Biomech (Bristol, Avon) 20(1): 70-76, 2005. PMID: 15567539. DOI: 10.1016/j.clinbiomech.2004.08.008

32 Peters CL, Bachus KN and Davitt JS: Fixation of periprosthetic femur fractures: a biomechanical analysis comparing cortical strut allograft plates and conventional metal plates. Orthopedics 26(7): 695-699, 2003. PMID: 12875564.

33 Dennis MG, Simon JA, Kummer FJ, Koval KJ and DiCesare PE: Fixation of periprosthetic femoral shaft fractures occurring at the tip of the stem: a biomechanical study of 5 techniques. J Arthroplasty 15(4): 523-528, 2000. PMID: 10884215. DOI: 10.1054/arth 2000.4339

34 O'Connell RS, Owen JR, Hansen EJ, Bashir AS, Wayne JS, Satpathy $J$ and Kates SL: Biomechanical evaluation of osteoporotic proximal periprosthetic femur fractures with proximal bicortical fixation and allograft struts. J Orthop Trauma 32(10): 508-514, 2018. PMID: 30247278. DOI: 10.1097/BOT. 0000000000001261 